UB-ECM-PF 92/12

\title{
Higher derivative quantum gravity with torsion in the conformally self-dual limit
}

\author{
E. Elizalde and S.D. Odintsovf \\ Department E.C.M., Faculty of Physics, \\ University of Barcelona, \\ Diagonal 647, 08028 Barcelona, Spain \\ e-mail:eli@ebubecm1.bitnet
}

May 11th, 1992

\begin{abstract}
The path integral for higher-derivative quantum gravity with torsion is considered. Applying the methods of two-dimensional quantum gravity, this path integral is analyzed in the limit of conformally self-dual metrics. A scaling law for fixed-volume geometry is obtained.
\end{abstract}

PACS:

03.70 Theory of quantized fields, 04.50 Unified theories and other theories of gravitation, 11.10 Field theory.

${ }^{1}$ On leave from Department of Mathematics and Physics, Pedagogical Institute, 634041 Tomsk, Russia. 
It is widely known that higher-derivative quantum gravity is multiplicatively renormalized [1] and that it is an asymptotically free theory [2]. It provides a very natural framework for a consistent theory of quantum gravity and for unification with matter. Unfortunately, in spite of the numerous efforts by different people [1-5], the unitarity of this theory is still an unsolved problem (for a general review of higher-derivative gravity and of GUTs interacting with higher-derivative gravity see [6]).

On the other side, it is known that higher-derivative quantum gravity with torsion can also be multiplicatively renormalized, and that it is unitary (free of ghost poles), at least for some values of the parameters involved an at the tree level [7]. It can be therefore interesting to study the properties of this theory on the quantum level in some detail, because it can provide a new framework for a consistent theory of quantum gravity. However, higher-derivative gravity with torsion [7] is very complicated - already at the classical level - and some reasonable simplifications must be introduced from the begining.

A slightly simplified version of the full theory, which has been discussed in [6], is described by the following Lagrangian:

$$
\begin{aligned}
L_{G} & =\lambda_{0}+\gamma_{0} R+\eta_{0} R^{2}+\rho_{0} F+\alpha_{01} F_{\mu \nu}^{2}+\alpha_{02}\left(\nabla_{\mu} S^{\mu}\right)^{2}+\alpha_{03}\left(S_{\mu} S^{\mu}\right)^{2} \\
& +\alpha_{04} R^{\mu \nu} S_{\mu} S_{\nu}+\alpha_{05} R S_{\mu} S^{\mu}+\gamma_{0} \alpha_{06} S_{\mu} S^{\mu}+\text { surface terms. }
\end{aligned}
$$

Here $F=C_{\mu \nu \alpha \beta}^{2}$ is the Weyl tensor, $R_{\mu \nu}$ the Ricci tensor, and $R$ the curvature, associated with $\nabla_{\mu}$ (the covariant derivative without torsion), $F_{\mu \nu}=\nabla_{\mu} S_{\nu}-\nabla_{\nu} S_{\mu}$, with $S^{\nu}=$ $i \epsilon^{\alpha \beta \mu \nu} T_{\alpha \beta \mu}$, being $T_{\alpha \beta \mu}$ the torsion tensor and, finally, $\lambda_{0}, \ldots, \alpha_{06}$, are the bare coupling constants of the theory.

We shall consider the case when only the antisymmetric part of the torsion (i.e., $S_{\mu}$ ) is non-zero, while all the other components of the torsion are zero. Notice that torsion can minimally interact with matter (spinors) only through the $S_{\mu}$. Another reason in favor of our restriction is the fact that the vector $S_{\mu}$ is most important for the cosmological applications of gravity with torsion.

A theory with the Lagrangian (1) is multiplicatively renormalized. The perturbative approach to this theory has not been developed yet (one may even question its existence), due to the presence of the vector field $S_{\mu}$, which is not the gauge field. We do not have a 
gauge-fixing term for it, and we do not even know how to construct the propagator.

The purpose of this letter is to show that some quantum elements and properties of the theory (1) - such as its path integral in the self-dual limit and some scaling lawscan be investigated. The path integral which is interesting for our analysis is the following (written in Euclidean coordinates)

$$
\int \mathcal{D} g_{\mu \nu} \mathcal{D} S_{\alpha} \mathcal{D} \psi \exp \left(-S_{m}\left[g, S_{\mu}, \psi\right]-\int_{\mathcal{M}} d^{4} x \sqrt{g} L_{G}\right) O_{1}\left[g, S_{\mu}, \psi\right] \cdots O_{n}\left[g, S_{\mu}, \psi\right],
$$

where $\psi$ is the set of matter fields, $S_{m}$ is the conformally invariant free matter action, $L_{G}$ is given by eq. (1), the manifold $\mathcal{M}$ has a fixed topology, and the $O_{i}, i=1, \ldots, n$, are some operators.

We now follow ref. [8], where the path integral (2) without torsion and in the self-dual limit $\rho_{0} \rightarrow \infty$ has been studied. Presumably, such limit describes the infrared dynamics of four-dimensional quantum gravity [9] (see also [10]). When $\rho_{0} \rightarrow \infty$, the path integral over $g_{\mu \nu}$ becomes the integral over conformally self-dual metrics. Notice that the delta function arising from the conformally self-dual part of the Weyl tensor reduces the integral over the 10 components of $g_{\mu \nu}$ to an integral over 5 components only [8].

Explicitly, the conformally self-dual metric has the form

$$
g_{0}=\left(\widehat{g}\left(m_{i}\right) e^{\Phi}\right)^{\xi}
$$

where $\widehat{g}\left(m_{i}\right)$ can be fixed via $\widehat{R}=0$ and $\partial^{\mu} \widehat{g}_{\mu \nu}=0$. The $m_{i}$ parametrize the moduli space of conformally self-dual metrics (see [11]) up to Weyl transformations, $g_{\mu \nu} \rightarrow g_{\mu \nu} e^{\Phi}$, and diffeomorphisms, $x^{\mu} \rightarrow x^{\mu}+\xi^{\mu}$ (see [8] for details). With the above choice,

$$
\delta g_{\mu \nu}=g_{0 \mu \nu} \delta \Phi+\nabla_{(\mu} \delta \xi_{\nu)}+\delta \bar{h}_{\mu \nu}
$$

In the limit $\rho_{0} \rightarrow \infty$, the integral over $\delta \bar{h}_{\mu \nu}$ can be calculated, with the following result $[8]$

$$
\begin{array}{r}
\int S_{\mu} \int \prod_{i} d m_{i} \mathcal{D} \Phi \operatorname{det}\left(O^{+} O\right)_{\widehat{g} e^{\Phi}}^{-1 / 2} \operatorname{det}\left(L^{+} L\right)_{\widehat{g} e^{\Phi}}^{1 / 2} \\
\times \quad \operatorname{det}\left(\square^{m a t}\left(S_{\mu}\right)\right)_{\widehat{g} e^{\Phi}}^{-1 / 2} \exp \left[-\left.\int d^{4} x \sqrt{g} L_{G}\right|_{\rho=0}\right],
\end{array}
$$


where the contribution of the matter partition function is $\operatorname{denoted}$ by $\operatorname{det}\left(\square^{\text {mat }}\right)^{-1 / 2}$ (of course, for spinors the sign of the exponent, $-1 / 2$, is reversed), and the number of degrees of freedom (spinors, gauge fields) should be also taken into account. Moreover, $\left(L^{+} L\right)_{\nu}^{\mu}=$ $-2\left(\delta_{\nu}^{\mu} \square+\frac{1}{2} \nabla^{\mu} \nabla_{\nu}+R_{\nu}^{\mu}\right) . O^{+} O$ is a conformally invariant fourth-order differential operator acting on tensors and coming from the expansion of $C_{\mu \nu \alpha \beta}^{2}$ (see $\left.[8,12]\right)$. Finally, the integral over the diffeomorphism group, $\mathcal{D} \xi$ has been dropped.

Let us concentrate now on the integral $\int \mathcal{D} \Phi$. To decouple the determinants from $\Phi$ we should look to the conformal anomaly of matter. The conformal matter action is given by $[6]^{2}$

$$
\begin{aligned}
S_{m} & =\int d^{4} x \sqrt{g}\left[\sum_{i} \varphi_{i}\left(\square-\frac{1}{6} R-\xi S_{\mu} S^{\mu}\right) \varphi_{i}\right. \\
& \left.+\sum_{j} i \bar{\psi}_{j}\left(\gamma^{\mu} \nabla_{\mu}-\zeta \gamma_{5} \gamma^{\mu} S_{\mu}\right) \psi_{j}\right] .
\end{aligned}
$$

Here $\xi$ and $\zeta$ are the constants of non-minimal coupling of matter with torsion, and the $\varphi_{i}$ and $\psi_{j}$ are the scalars and spinors, respectively. Note that the values $\xi=0, \zeta=1 / 8$ correspond to the minimal interaction of matter with torsion. (In two dimensions matter does not interact minimally with torsion). Note also that the action of gauge fields is the same as in the absence of torsion.

The trace anomaly has the following form [6]

$$
\begin{aligned}
<T_{\mu}^{\mu}> & =\frac{2}{\sqrt{g}} \frac{\delta S\left[\widehat{g}, \Phi, S_{\mu}\right]}{\delta \Phi}=-\frac{1}{(4 \pi)^{2}}\left[a\left(F-\frac{2}{3} \square R\right)+b G\right. \\
& \left.+a_{1} F_{\mu \nu}^{2}+a_{2}\left(S_{\mu} S^{\mu}\right)^{2}+a_{3} \square\left(S_{\mu} S^{\mu}\right)+a_{4} \nabla_{\mu}\left(S_{\nu} \nabla^{\nu} S_{\mu}-S^{\mu} \nabla_{\nu} S^{\nu}\right)\right] \\
& +\lambda^{\prime}+\gamma^{\prime} R+\gamma^{\prime} \alpha^{\prime}{ }_{6} S_{\mu} S^{\mu}+\alpha^{\prime}{ }_{2}\left(\nabla_{\mu} S^{\mu}\right)^{2}+\alpha^{\prime}{ }_{4} R^{\mu \nu} S_{\mu} S_{\nu}+\alpha^{\prime}{ }_{5} R S_{\mu} S^{\mu}+b^{\prime} \square R
\end{aligned}
$$

where the values (finite) of $a$ and $b$ are well known (see, for example, [12]), and [6]

$$
a_{1}=a_{4}=-\frac{2}{3} \zeta^{2}, \quad a_{2}=\frac{1}{2} \xi^{2}, \quad a_{3}=\frac{1}{3}\left(2 \zeta^{2}-\frac{1}{2} \xi^{2}\right) .
$$

The divergent parameters $\alpha^{\prime}, \gamma^{\prime}, \ldots, b^{\prime}$, renormalize the corresponding coupling constants.

\footnotetext{
${ }^{2}$ For a detailed (non-trivial) discussion of the real and imaginary parts of the euclidean matter action in the (more simple) case of constant background fields see [13].
} 
Using the equation which is valid for conformally invariant differential operators

$$
\operatorname{det} X_{\widehat{g} e^{\Phi}}=\operatorname{det} X_{\widehat{g}} \exp \left(-S\left[\widehat{g}, \Phi, S_{\mu}\right]\right)
$$

where $X_{\widehat{g}}$ can depend on $S_{\mu}\left(\widehat{S}_{\mu}=S_{\mu}\right)$, if $X_{\widehat{g} e^{\Phi}}$ depends on $S_{\mu}$, and integrating over the trace anomaly (7) [14] (see also $[15,9]$ ) for the case $S_{\mu}=0$ ), we can rewrite (5) as follows

$$
\int \mathcal{D} S_{\mu} \int \prod_{i} d m_{i} \chi\left(m_{i}, S_{\mu}\right) \int \mathcal{D} \Phi \exp \left(-S\left[\widehat{g}, \Phi, S_{\mu}\right]\right),
$$

where

$$
\chi\left(m_{i}, S_{\mu}\right)=\operatorname{det}\left(O^{+} O\right)_{\widehat{g}\left(m_{i}\right)}^{-1 / 2} \operatorname{det}\left(L^{+} L\right)_{\widehat{g}\left(m_{i}\right)}^{1 / 2} \operatorname{det}\left(\square^{m a t}\left(S_{\mu}\right)\right)_{\widehat{g}\left(m_{i}\right)}^{-1 / 2}
$$

depends also on the torsion $S_{\mu}$, due to the interaction of matter with torsion. We have

$$
\begin{aligned}
S\left[\widehat{g}, \Phi, S_{\mu}\right] & =\frac{B_{0}}{32 \pi^{2}} S_{0}[\widehat{g}, \Phi]+\frac{A_{0}}{32 \pi^{2}} S_{1}[\widehat{g}, \Phi]+\eta_{1} S_{R^{2}} \\
& +\gamma_{1} S_{R}+\lambda_{1} S_{c c}+\left(\alpha_{1}+\frac{a_{1}}{(4 \pi)^{2}}\right) S_{F}+\left(\alpha_{3}+\frac{a_{2}}{(4 \pi)^{2}}\right) S_{4} \\
& +\frac{1}{(4 \pi)^{2}}\left(a_{3}+\frac{1}{2} a_{4}\right) S_{2}+\frac{a_{4}}{(4 \pi)^{2}} S_{21}+\cdots
\end{aligned}
$$

Here

$$
\begin{aligned}
& A_{0}=a_{0}+a_{L}+a_{m a t}, \quad B_{0}=b_{0}+b_{L}+b_{\text {mat }}, \\
& S_{0}[\widehat{g}, \Phi]=\int d^{4} x \sqrt{\widehat{g}}\left[\Phi \Delta \Phi+\left(\widehat{G}-\frac{2}{3} \square \widehat{R}\right) \Phi\right], \\
& \Delta=\square^{2}+2 \widehat{R}^{\mu \nu} \widehat{\nabla}_{\mu} \widehat{\nabla}_{\nu}-\frac{2}{3} \widehat{R} \square+\frac{1}{3 ? ? ?}(\widehat{\nabla} \mu \widehat{R}) \widehat{\nabla_{\mu}}, \\
& S_{1}[\widehat{g}, \Phi]=\int d^{4} x \sqrt{\widehat{g}} \widehat{F} \Phi, \\
& S_{R^{2}}=\int d^{4} x \sqrt{\widehat{g}}\left[\widehat{R}-\frac{3}{2}(\widehat{\nabla} \Phi)^{2}-3 \square \Phi\right]^{2}, \\
& S_{R}=\int d^{4} x \sqrt{\widehat{g}}\left[\widehat{R}-\frac{3}{2}(\widehat{\nabla} \Phi)^{2}-3 \square \Phi\right], \\
& S_{c c}=\int d^{4} x \sqrt{\widehat{g}} e^{2 \Phi}, \quad S_{F}=\frac{1}{2} \int d^{4} x \sqrt{\widehat{g}} \widehat{F}_{\mu \nu}^{2} \Phi, \\
& S_{4}=\frac{1}{2} \int d^{4} x \sqrt{\widehat{g}} \widehat{S}_{\mu}^{4} \Phi, \quad S_{2}=\frac{1}{4} \int d^{4} x \sqrt{\widehat{g}} \widehat{S}^{2} \widehat{\nabla}_{\mu} \Phi \widehat{\nabla}^{\mu} \Phi, \\
& S_{21}=\frac{1}{4} \int d^{4} x \sqrt{\widehat{g}} \widehat{S}^{\mu} \widehat{S}^{\nu} \widehat{\nabla}_{\mu} \Phi \widehat{\nabla}_{\nu} \Phi .
\end{aligned}
$$

Here $\widehat{S}_{\mu}=S_{\mu}$, and $a_{0}, b_{0}, a_{L}$ and $b_{L}$ are the contributions from $O^{+} O$ and $L^{+} L$ to the trace anomaly. 
We do not write explicitly in (12) the terms dependent on torsion in which there is no finite contribution from the conformal anomaly. (We will be interested only in the critical point, where the corresponding renormalized coefficients in (1), i.e., $\alpha_{2}, \alpha_{4}, \alpha_{5}$ and $\alpha_{6}$ are equal to zero). Note also that the $\Phi$-independent terms have been omitted in (12). It is interesting to notice that torsion in (12) has appeared in a much more complicated way than in two dimensions, where it can be integrated over. Here we cannot even decouple the $\Phi$ and $S_{\mu}$ terms in the path integral.

Now, the measure $\mathcal{D}_{\widehat{g} e^{\Phi}} \Phi$ can be treated as in two dimensions [16], and the $\Phi$-dependence can be absorbed in the coefficients of the gravitational part of $(12)\left(S_{\mu}=0\right)$, as it has been done in [8]. It changes the coefficients $A$ and $B$, due to the contribution of the operator $\Delta$ :

$$
\begin{aligned}
A & =\frac{1}{120}\left(N_{0}+6 N_{1 / 2}+12 N_{1}-8\right)+\frac{199}{30} \\
B & =-\frac{1}{360}\left(N_{0}+11 N_{1 / 2}+62 N_{1}-28\right)-\frac{87}{20}
\end{aligned}
$$

where the last term in both brackets is the contribution of $\Delta$ to the conformal anomaly $[10,12]$, and the last terms in (14) and (15) are the $a_{0}+a_{L}$ and $b_{0}+b_{L}$ found in [12], respectively. These contributions are equal to the trace anomaly coefficients of conformal quantum gravity' (see ref. [2]).']

The additional contributions to the terms of conformal anomaly connected with torsion are given only by the $\Delta$-operator (which accounts for the torsionic terms from (13)). The direct calculation we have done using the algorithm of fourth order operator divergencies evaluation, gives for the coefficient which is of interest to us:

$$
a_{2}^{(\Delta)}=\frac{1}{4 B_{0}^{4}}\left[\frac{1}{2} a_{3}^{2}+\frac{3}{4} a_{3} a_{4}+\frac{5}{16} a_{4}^{2}\right] .
$$

Here, $B_{0}(15)$ is given by the matter part only (the first three terms in (15)).

\footnotetext{
${ }^{3}$ This fact also ensures the validity of the so-called conformal regularization procedure [17] in conformal Weyl gravity.

${ }^{4}$ It would be interesting to understand whether the coefficients $A$ and $B$ (i.e., their signs) can be changed in some other conformal gravity model. For example, for the second-order conformal gravity of [18] (i.e., $O^{+} O$ is of second order), we have $b_{0}+b_{L}=-27 / 20$. Surely, the signs of $A$ and $B$ can be changed in conformal supergravity (second ref. [15]).
} 
With $A$ and $B$ known, one can start the calculation of the vertex operators. For example, we can look to the scaling (classical plus anomalous) dimension of $e^{\alpha \Phi}$ corresponding to $S_{c c}=\int d^{4} x \sqrt{\widehat{g}} e^{2 \alpha \Phi}$ and find it in terms of $B[8]$. We can also look to other gravitational [10] or torsion vertex operators.

Finally, we will try to define the scaling law in the presence of torsion. Starting from the fixed-volume partition function at the critical point (as in [8], $\eta_{1}=\gamma_{1}=\lambda_{1}=0$ and the torsionic coupling constants where there is no contribution from the conformal anomaly are also equal to zero), we get

$$
\begin{aligned}
& \int d S_{\mu} Z\left(V, S_{\mu}\right)=\int \mathcal{D} S_{\mu} \int \prod_{i} d m_{i} \chi\left(m_{i}, S_{\mu}\right) \int \mathcal{D}_{\widehat{g}} \Phi \\
\times & \exp \left\{-\frac{B}{32 \pi^{2}} S_{0}[\widehat{g}, \Phi]-\frac{A}{32 \pi^{2}} S_{1}[\widehat{g}, \Phi]-\frac{1}{(4 \pi)^{2}}\left(a_{3}+\frac{1}{2} a_{4}\right) S_{2}\left[\widehat{g}, \Phi, S_{\mu}\right]\right. \\
- & \left.\frac{a_{4}}{(4 \pi)^{2}} S_{21}\left[\widehat{g}, \Phi, S_{\mu}\right]-\left(\alpha_{1}+\frac{a_{1}}{(4 \pi)^{2}}\right) S_{F}-\left(\alpha_{3}+\frac{a_{2}}{(4 \pi)^{2}}\right) S_{4}\right\} \delta\left(\int d^{4} x \sqrt{\widehat{g}} e^{2 \alpha \Phi}-V\right) .
\end{aligned}
$$

Using the properties of the exponential in (13) under the constant shift $\Phi \rightarrow \Phi+c$, we get

$$
Z\left(V, S_{\mu}^{2}\right)=\exp \left\{\left[-2 \alpha-B \chi+\frac{3}{2} A \tau-\left(\alpha_{3}+\frac{a_{2}}{(4 \pi)^{2}}\right) \bar{S}_{4}\right] c\right\} Z\left(e^{-2 \alpha c} V, S_{\mu}^{2}\right),
$$

where $\chi$ is the Euler characteristic of the manifold, $\tau$ its signature, and $\bar{S}_{4}=\frac{1}{2} \int d^{4} x \sqrt{\hat{g}} S_{\mu}^{4}$. It follows from (17), that

$$
Z\left(V, S_{\mu}^{2}\right) \sim \exp \left\{\left[-1+\frac{1}{4 \alpha}\left(-2 B \chi+3 A \tau-2\left(\alpha_{3}+\frac{a_{2}}{(4 \pi)^{2}}\right) \bar{S}_{4}\right)\right] \ln V\right\} .
$$

The scaling law (18) can be the starting point for computer simulations of the theory. Of course, one can look to other scaling laws corresponding to fixed torsion.

In conclusion, we have discussed here the path integral corresponding to higher derivative quantum gravity with torsion in the self-dual limit, generalizing the work [8]. We have found that torsion actually shows up in a very complicated way, and that it cannot be integrated over, as in two dimensions [19]. Torsion does not decouple from the conformal factor in this formalism. Of course, one can guess that using some other variables (as, for example, vielbein and connection) the situation could be improved. 
Finally, the above approach can be critized on general grounds, because even the classification of four-dimensional manifolds is not known (if it at all exists), and here we cannot expect that the methods of two-dimensional quantum gravity can be very useful in four dimensions. Nevertheless, our point is that already the fact that these methods can be applied (as we have shown) in four dimensions for the discussion of some limiting cases of the theory (1) - which probably cannot be formulated at all at the perturbative level—actually seems very promising.

\section{Acknowledgments}

We would like to thank Profs. Ignatios Antoniadis and Rolf Tarrach for fruitful discussions on related problems. S.D.O. thanks the members of the Department E.C.M. of Barcelona University for the kind hospitality. E.E. thanks the Alexander von Humboldt Foundation for continued logistic help. This work has been supported by Direcci"n Ge-

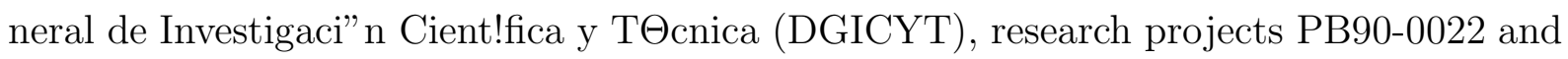
SAB92-0072. 


\section{References}

[1] K.S. Stelle, Phys. Rev. D16 (1977) 953; Gen. Rel. Grav. 9 (1978) 353.

[2] E.S. Fradkin and A.A. Tseytlin, Nucl. Phys. B201 (1982) 469.

[3] A. Salam and S. Strathdee, Phys. Rev. D18 (1978) 4480.

[4] T. Antoniadis and E.T. Tomboulis, Phys. Rev. D33 (1986) 2756.

[5] S.W. Hawking, in Quantum Field Theory and Quantum Statistics, eds. I.A. Batalin, C.J. Isham and G.A. Vilkovisky (Hilger, Bristol, I987).

[6] I.L. Buchbinder, S.D. Odintsov and I.L. Shapiro, Effective Action in Quantum Gravity (Hilger, Bristol, 1992); see also Rivista Nuovo Cim. 12 (1989) 1.

[7] E. Sezgin and P. van Nieuwenhuizen, Phys. Rev. D21 (1980) 3269.

[8] C. Schmidhuber, preprint CALTECH, 1992.

[9] I. Antoniadis and E. Mottola, Phys. Rev. D45 (1992) 2013.

[10] S.D. Odintsov, Z. Phys. C, 1992, in press.

[11] T. Eguchi, P.B. Gilkey and A.J. Hanson, Phys. Rep. 66 (1980) 213.

[12] I. Antoniadis, P.O. Mazur and E. Mottola, preprint LA-UR-92, 1992.

[13] E. Elizalde, Phys. Lett. 145B (1984) 271.

[14] I.L. Buchbinder, S.D. Odintsov and I.L. Shapiro, Phys. Lett. B162 (1985) 193.

[15] R.J. Reigert, Phys. Lett. B134 (1984) 56; E.S. Fradkin and A.A. Tseytlin, Phys. Lett. 134 (1984) 187; E.T. Tomboulis, Nucl. Phys. B329 (1990) 410; S.D. Odintsov and I.L. Shapiro, Class. Quant. Grav. 8 (1991) L57.

[16] V. Knizhnik, A.M. Polyakov and A.B. Zamolodchikov, Mod. Phys. Lett. A3 (1988) 819; J. Distler and H. Kawai, Nucl. Phys. B321 (1989) 509; F. David, Mod. Phys. Lett. A3 (1988) 1651. 
[17] F. Englert, C. Truffin and R. Gastmans, Nucl. Phys. B117 (1976) 407; E.S. Fradkin and G.A. Vilkovisky, Phys. Lett. B73 (1978) 209.

[18] P. Pascual, J. Taron and R. Tarrach, Phys. Rev. D39 (1989) 2993.

[19] T. Berger and I. Tsutsui, Nucl. Phys. B333 (1990) 245. 